\title{
Pulsar Wind Nebulae and Cosmic Rays: A Bedtime Story
}

\author{
A. Weinstein for the VERITAS Collaboration
}

\begin{abstract}
The role pulsar wind nebulae play in producing our locally observed cosmic ray spectrum remains murky, yet intriguing. Pulsar wind nebulae are born and evolve in conjunction with SNRs, which are favored sites of Galactic cosmic ray acceleration. As a result they frequently complicate interpretation of the gamma-ray emission seen from SNRs. However, pulsar wind nebulae may also contribute directly to the local cosmic ray spectrum, particularly the leptonic component. This paper reviews the current thinking on pulsar wind nebulae and their connection to cosmic ray production from an observational perspective. It also considers how both future technologies and new ways of analyzing existing data can help us to better address the relevant theoretical questions. A number of key points will be illustrated with recent results from the VHE $(\mathrm{E}>100 \mathrm{GeV})$ gamma-ray observatory VERITAS.
\end{abstract}

Keywords:

\section{Introduction}

The discovery of cosmic rays in the early twentieth century $[1,2]$ inaugurated a decades-long, serialized mystery story that has yet to be completed. At the heart of the mystery lies a set of of simple questions. What particles appear in the cosmic ray spectrum that we see from Earth? Where do these particles originate? How are they accelerated? In recent decades direct cosmic ray observations have brought us a wealth of information on the cosmic ray spectrum and its composition, clues to the types of environments in which cosmic rays are born. The interaction of these particles with interstellar magnetic fields, however, prevents them from being traced back to their point of origin.

In order to pursue the other half of this mystery-the nature of cosmic ray accelerators-we must use the secondary photon radiation produced in these cosmic ray nurseries. These high-energy (300 MeV - $100 \mathrm{GeV}$ ) and very high-energy (VHE; $E>100 \mathrm{GeV}$ ) gamma rays are not deflected by interstellar magnetic fields and can be used to map cosmic ray populations in and near their parent accelerators. The different processes by which relativistic cosmic ray electrons, protons, and heavier nuclei produce high-energy gamma rays leave characteristic imprints on the gamma-ray energy spectrum of particular astrophysical accelerators. These features may be used to constrain the composition and energy spectrum of accelerated particle populations.

The local cosmic ray spectrum is known to be a mix of protons and heavier nuclei, with a smaller admixture of leptons. A mix of astrophysical accelerators within and outside our Galaxy is believed to contribute, including shocks arising in supernova remnants (SNRs), shocks formed by interacting high-velocity winds from massive stars [3], pulsars, and active galactic nuclei (AGN). We focus here on a single subplot of a much larger story - the role played by pulsar wind nebulae (PWNe) in our quest to understand the Galactic cosmic ray spectrum.

\section{Dramatis Personae}

No single gamma-ray observatory provides a complete picture of the gamma-ray sky at all energies and spatial scales. The Fermi Gamma-ray Space Telescope (Fermi LAT) can map the entire gamma-ray sky between $300 \mathrm{MeV}$ and $500 \mathrm{GeV}$ over all spatial scales, with an angular resolution that ranges from $0.1^{\circ}$ to $1^{\circ}$ depending 


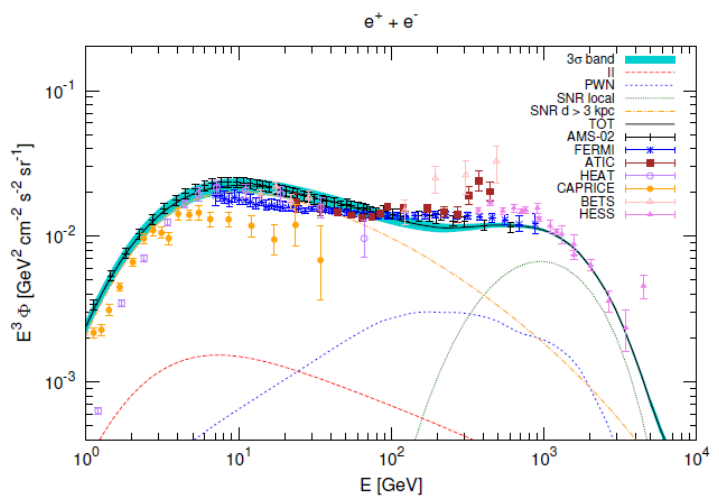

Figure 1: Comparison to extant data from AMS-02 [4], Fermi LAT [5], ATIC [6], HEAT [7], CAPRICE[8], BETS[9], and H.E.S.S.[10] of a model considering contributions to the $e^{ \pm}$ flux from the following categories: $e^{-}$from distant $(>3 \mathrm{kpc}$ ) SNRs (dot-dashed yellow) and local SNRs (dotted green), secondary $e^{ \pm}$(long dashed red) and $e^{ \pm}$from PWNe (short dashed blue). The model is derived from a simultaneous fit to all AMS-02 data. Reproduced from [11]. (A color version of this figure is available in the online journal).

on energy. However, Fermi LAT data become photonpoor above $10 \mathrm{GeV}$. Efficient detection of gamma rays above $100 \mathrm{GeV}$ requires ground-based gamma-ray observatories. Arrays of imaging atmospheric Cherenkov telescopes (IACTs), treat the atmosphere as the sensitive volume of an electromagnetic calorimeter. Sampling the pool of Cherenkov light produced by atmospheric showers from multiple views permits better reconstruction of the arrival direction and energy of the primary gamma ray [12]. Currently-operating IACT arrays include VERITAS and MAGIC in the northern hemisphere and H.E.S.S. in the southern hemisphere. These instruments have fields of view between $2^{\circ}$ and $5^{\circ}$. While the precise energy threshold varies by observatory, all are sensitive between $100 \mathrm{GeV}$ and $10 \mathrm{TeV}$. RPC carpet detectors such as ARGO-YBJ [13] and water Cherenkov detectors, such as the recently-decommissioned Milagro [14, 15] and the newly-commissioned HAWC [16] have wide fields of view and the best access to energies above $10 \mathrm{TeV}$, effectively complementing the IACTs.

\subsection{VERITAS}

VERITAS, an array of four 12-m IACTs in southern Arizona, is currently the most sensitive TeV gamma-ray instrument in the northern hemisphere. Each of the four cameras has a $3.5^{\circ}$-diameter field of view (giving the array an overall field of view of $3.5^{\circ}$ ) and is equipped with 499-pixel photomultiplier tube camera. VERITAS has an angular resolution (68\% containment) of better than $0.1^{\circ}$ at $1 \mathrm{TeV}$ and is sensitive to sources with integral fluxes less than $1 \%$ of the Crab Nebula flux with less than 30 hours of observation time. VERITAS has been in continuous operation as a four-telescope array since January 2007. It underwent a series of upgrades in the period between 2009-2012 that increased the instrument's sensitivity [17] and decreased its energy threshold.

\subsection{Pulsar Wind Nebulae}

The rapidly spinning neutron stars known as pulsars are thought to be some of the most powerful sources of electrons and positrons $\left(e^{ \pm}\right)$in the Galaxy [11]. The pulsar is thought to possess a strong, rotating magnetic field that generates a powerful electric field, which then rips particles free from the pulsar surface. Once accelerated, these charged particles can produce further particleantiparticle pairs through curvature radiation, creating a relativistic charged-particle wind. Since the pulsar is created by a supernova explosion, both the pulsar and its wind are initially located within the SNR. The interaction of the pulsar wind with the slower supernova ejecta creates a termination shock. The magnetized, relativistic plasma between the termination shock and the ejecta is known as the pulsar wind nebula (PWN). Particles and antiparticles are accelerated to very high energies before becoming trapped by the PWN magnetic field. Over time the PWN magnetic field weakens and these particles are injected into the interstellar medium (ISM).

\section{Pulsar Wind Nebulae and the Positron Excess}

The origin of cosmic ray electrons and positrons, like the origin of the protons and heavier nuclei that make up the dominant portion of the terrestrial cosmic ray spectrum, is a question of great interest. Typical models of the cosmic ray lepton spectrum invoke the following contributions, as illustrated in Figure 1: electrons produced in SNRs and secondary electrons and positrons from spallation reactions of hadronic cosmic rays with the ISM. The secondary $e^{ \pm}$components are expected to fall off quickly above $100 \mathrm{GeV}$; distant SNRs also contribute strongly to the electron spectrum below this energy. Electrons produced in local SNRs are expected to fill out the electron spectrum above $100 \mathrm{GeV}$. In this picture the fraction of positrons in the cosmic ray lepton spectrum should decrease with increasing energy, in contradiction to results from ATIC [6], PAMELA [18], 
Fermi LAT [19] and AMS [4], which show that the positron fraction in fact increases with energy. While this effect could be produced by WIMP dark matter annihilation, the anomalous positron fraction can also be explained by nearby astrophysical sources, most notably pulsars and PWNe.

$e^{ \pm}$accelerated in the magnetosphere of a mature pulsar are less likely to be confined by an associated PWN. They have consequently been advanced as a viable explanation of the positron fraction excess [20, 21]. Most recently, Di Mauro et al. [11] have used AMS-02 data to constrain pulsar/PWN origin scenarios for the positron excess. As AMS-02 provides separate measurements of the electron, positron, and all-lepton spectra as well as the positron fraction, these data provide the largest set of simultaneous constraints. Di Mauro et al. report that these data can accommodate the positron excess using a contribution from either the entire pulsar catalog, a few ( $\sim$ ) of the brightest local $(d<1 \mathrm{kpc})$ pulsars with ages less than $3000 \mathrm{kyr}$, or a single local pulsar/PWN. There are only three single-source candidates for which the fit returns a physically reasonable requirement for the emitted power. Of these, Di Mauro et al. strongly favor Geminga, a 340 kyr old X-ray and gamma-ray pulsar with a compact $\left(\sim 2^{\prime}\right)$ X-ray PWN [22], since it is the only source for which the $e^{ \pm}$emission efficiency is not required to be close to one [11].

Others have also noted that the nearby Geminga pulsar could account for the positron excess on its own. Yüksel, Kislev, and Stanev, in particular, point to Milagro's detection of $>20 \mathrm{TeV}$ gamma-ray emission (MGRO J0634.0+1745) in a $2.6^{\circ}$ by $2.6^{\circ}$ region surrounding the pulsar as evidence for the production, acceleration and escape of $e^{ \pm}$with energies up to $100 \mathrm{TeV}$ [23]. These authors note that more detailed constraints on the spectrum and morphology of the $\mathrm{TeV}$ source are vital to constraining the particle population. They also anticipate a gamma-ray halo that extends into the energy range visible to IACTs and note that the halo at these energies provides essential information about the total energetics. For example, observing the gammaray spectrum of the halo soften with increasing distance from the pulsar would provide strong evidence for $e^{ \pm}$ cooling.

However, so far our picture of this nebula between $\mathrm{X}$-ray and multi-TeV energies remains blank. Fermi $L A T$ has only published upper limits on the flux from the PWN [24]. VERITAS likewise has no confirmed detection of the Geminga PWN $[25,26]$. In both cases this gap in our knowledge can be directly attributed to limitations of the instruments and data analysis methods used. With Fermi LAT data, separating the much
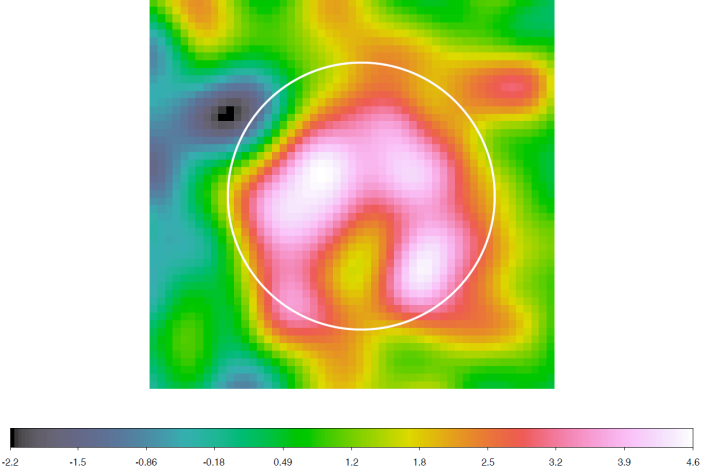

Figure 2: Comparison of the VERITAS field of view (white circle), centered at the position of the Geminga pulsar to the morphology of MGRO J0634.0+1745.

weaker PWN emission from that of the extremely bright pulsar is challenging and the methods used to do solooking at higher energies and looking only at off-pulse emission-reduce the available photon statistics. In the case of IACT observatories, the main difficulty is illustrated by Figure 2. Even for VERITAS, which has the most generous field of view of all IACTs in the northern hemisphere, MGRO J0634.0+1745 saturates the field of view. Standard data-analysis methods such as the reflected-region model and ring-background model [27] rely on portions of the field of view distant from the gamma-ray source to estimate the level of irreducible cosmic ray background. These methods break down when a source fills a large fraction the field of view.

\section{Pulsar Wind Nebulae in a Cosmic Ray Labora- tory}

Motivated in part by the hard spectrum of the very high-energy gamma-ray emission seen from the Vela X PWN [28, 29], some theorists have advanced hybrid PWNe models, in which the induced electric field extracts both $e^{ \pm}$pairs and heavy nuclei (e.g. iron) from the neutron star surface $[30,31,32]$. In this picture, the pulsar wind can play a significant role in accelerating these heavy nuclei and protons resulting from their photo-disintegration, both of which are eventually injected into the interstellar medium [30, 32]. These models, coupled with the ubiquity of TeV gamma-rayemitting PWNe [33, 34], suggest pulsars/PWNe as a (perhaps the only) source of Galactic cosmic rays above the knee $[30,32]$. This is consistent with measurements that indicate a heavier cosmic ray mass composition 
above the knee [35].

Moreover, as products of stellar death, pulsars, PWNe, and SNRs are born and evolve in concert. As a result, PWNe present as a complicating factor when trying to map regions of cosmic ray acceleration and detect signatures of cosmic ray escape. In the absence of unique spectral signatures, it is often unclear whether high and very high-energy gamma-ray emission should be attributed to particles accelerated within a SNR, or to accelerated leptons in some nearby PWN seen in the radio, X-ray, or optical. GeV gamma-ray emission from some bright pulsars (particularly those with significant off-pulse emission) can also be difficult to separate completely from the emission produced by a surrounding remnant.

Moreover, no one gamma-ray observatory currently in operation can provide the best determination of a source's gamma-ray spectrum at all energies, nor are these instruments necessarily well-matched in angular resolution. Milagro in particular has a best angular resolution of $0.5^{\circ}$ at $10 \mathrm{TeV}$ and worse than $1.5^{\circ}$ below $2 \mathrm{TeV}$. Consequently, contributions from multiple gamma-ray sources, the majority of which are actual or potential PWNe [36], are commingled in key energy ranges with the gamma-ray emission thought to arise from actively or recently accelerated (hadronic) cosmic ray populations. The inability to separate these sources, particularly at the highest energies, makes it difficult to establish the definitive broadband spectra needed constrain cosmic ray acceleration and escape models.

Nowhere are these challenges better illustrated than in the portion of the Galactic Plane that contains the Cygnus-X star-forming region ("Cygnus"). This region provides a superb laboratory for studying the early phases of the cosmic ray life cycle. It is the richest starforming region within $2 \mathrm{kpc}$ of Earth, with a total mass in molecular gas that is at least ten times that of all other nearby star-forming regions combined [38]. It contains a wealth of massive stars in stellar nurseries, young open clusters, and $\mathrm{OB}$ associations, the most massive of which, Cyg OB2, has been the subject of decades of focused observations by gamma-ray observatories [39]. However, the region's very richness lends itself to the types of source confusion discussed above. Moreover, observations of Cygnus provide a tangential view of the local arm, superimposing structures at many distances along the line of sight [38]. Conclusively establishing relationships between sources therefore relies on knowing the distance to each source, but these distances are in many cases poorly constrained or entirely unknown.

Two areas of Cygnus are of particular interest. One region surrounds hard-spectrum, spatially extended gamma-ray emission observed by Fermi LAT above 3 GeV (1FHL J2028.6+4110e) [37, 40] that is interpreted as a cocoon of cosmic rays. This region also contains Cyg OB2 and the radio and X-ray SNR G78.2+2.1 $[38,41,42]$. The second region surrounds the OB association Cyg OB1.

\subsection{The Cygnus Cocoon and its Vicinity}

The Cygnus cocoon covers an elongated region roughly four square degrees in size and fills a cavity carved in the ISM by stellar winds from a nearby grouping of OB stars. The hardness of the gamma-ray spectrum between $3 \mathrm{GeV}$ and $500 \mathrm{GeV}$ indicates that the generating cosmic ray spectrum is also hard, as it would be if the cosmic rays filling the cocoon were freshly accelerated $[37,40]$.

Determining the precise behavior of the cocoon spectrum at energies above $1 \mathrm{TeV}$ is critical if we are to correctly establish the nature and age of the accelerated particles that fill the cocoon. The cocoon is co-located with an extended source of $>20 \mathrm{TeV}$ gamma rays seen by Milagro (MGRO J2031+41) [43, 44]. While it is tempting to use the Milagro result to constrain the behavior of the cocoon spectrum at higher energies, the relationship between these two objects is not so straightforward. Viewed in gamma rays between a few $\mathrm{GeV}$ and $20 \mathrm{TeV}$, the region surrounding the cocoon is rife with sources, including at least one PWN and a SNR. As illustrated in Figures 3 and 5, all or part of the emission from these sources may contribute to the MGRO J2031+41 spectrum. Moreover, while Ackermann et al. [37] argue that the collective action of winds of massive stars are responsible for accelerating the cocoon particles, contributions from nearby phenomena such as SNR G78.2+2.1 and the Cyg OB2 association cannot be completely ruled out.

\subsection{SNR G78.2+21.1 and VER J2019+407}

SNR G78.2+2.1 (the $\gamma$ Cygni SNR) is a nearby $(\sim 1.7$ kpc) shell-like radio and X-ray SNR $\sim 1^{\circ}$ across ( $[41,42])$. The radio and $\mathrm{X}$-ray emission show higherintensity features to the north and south $[45,46]$. At $\sim 7000$ years $[41,47,42]$ it is on the young side of middle age. It appears to be in an early phase of adiabatic expansion into a low-density medium.

The center of the $\gamma$ Cygni SNR hosts a lowluminosity gamma-ray pulsar, PSR J2021+4026, which may or may not be the remnant of the $\gamma$ Cygni SNR's progenitor star [50, 51, 52]. Fermi LAT also observes hard (spectral index $2.39 \pm 0.14$ ) emission between $10 \mathrm{GeV}$ and $500 \mathrm{GeV}$ from the entire remnant 


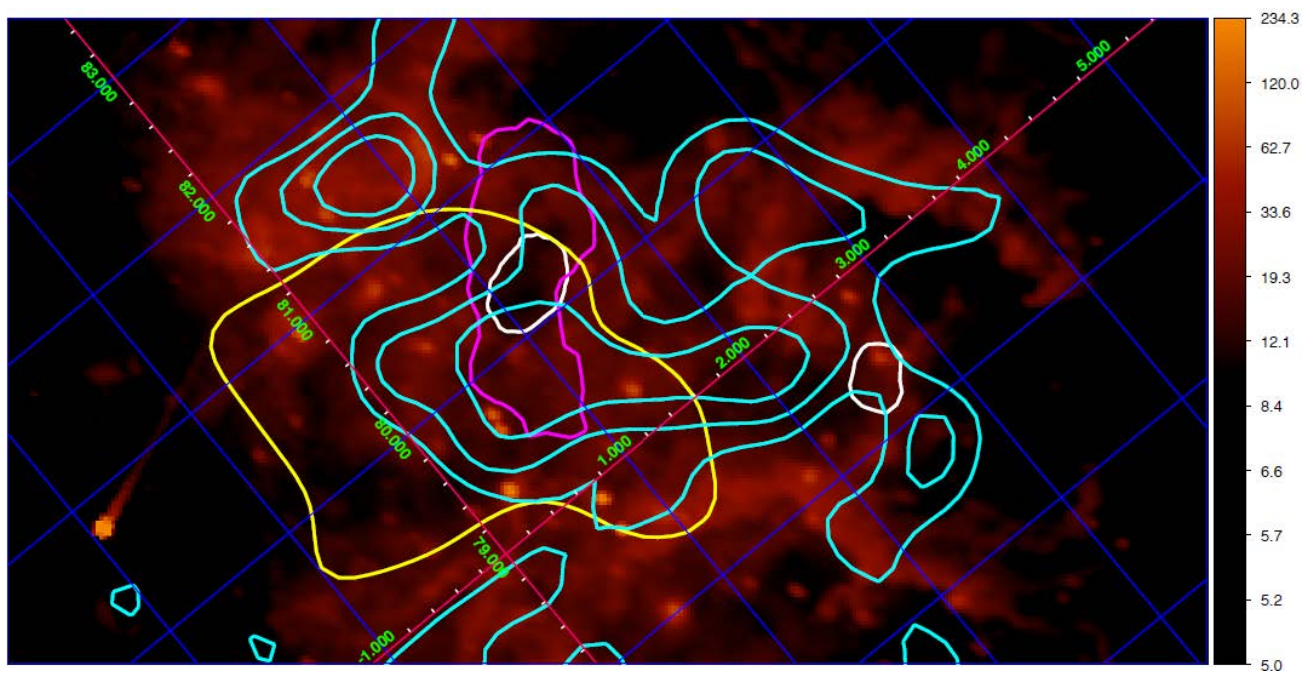

Figure 3: MSX $8 \mu \mathrm{m}$ infrared survey (color, $\mathrm{Wm}^{-2} \mathrm{sr}^{-1}, \log$ scale) for the vicinity of the Cygnus cocoon, showing the cavity in the interstellar medium. Fermi LAT 0.16, 0.24 and 0.32 photons bin ${ }^{-1}$ contours for the cocoon are shown in cyan [37]. 5 standard deviation contours for MGRO J2031+41, ARGO J2031+41, and the VERITAS sources are shown in yellow, pink, and white respectively. (A color version of this figure is available in the online journal).

$[37,40]$. VERITAS, by contrast, sees a more compact region of emission, VER J2019+407, coincident with the brightest part of the northern radio shell [36]. VER J2019+407's nature and relationship to the emission detected by Fermi LAT below $500 \mathrm{GeV}$ from the $\gamma$ Cygni SNR remains unclear. It is highly plausible that VER J2019+407 originates from protons and heavier nuclei accelerated in the SNR shock, but this scenario raises a number of questions. First, the compactness of VER J2019+407 is puzzling, given that high-intensity radio features are visible in both the north and south and the only indications of molecular material in the portion of the shell opposite to VER J2019+407. Furthermore, a reasonable power-law extrapolation of the Fermi LAT source up to $1 \mathrm{TeV}$ suggests that VERITAS should in principle see emission from the majority of the SNR.

This puzzle admits at least two competing physical solutions. The measured spectra of VER J2019+407 and the emission seen by Fermi LAT are both completely consistent with a power law with $\Gamma=2.4$. However, the spectra of different regions of the $\gamma$ Cygni SNR may evolve differently above $500 \mathrm{GeV}$, with the emission from the northern shell having a higher-energy cutoff than the remainder of the remnant. This portion of the remnant would consequently appear brighter at higher energies. The effect could be accentuated by systematic effects in the data analysis arising from the size of the $\gamma$ Cygni SNR and the presence of a magnitude-2 star overlapping the southeastern portion of the shell. Such effects would dilute VERITAS' sensitivity to emission from the entire SNR. VER J2019+407 may also be a chance superposition of a PWN in the the $\gamma$ Cygni SNR line of sight. While this interpretation is not favored, it has recently been lent some weight by the detection of a nearby hard X-ray point source [53].

\subsection{TeV J2032+4130 and Cyg OB2}

The serendipitous detection of the gamma-ray source $\mathrm{TeV} \mathbf{J} 2032+4130$, based on observations made by the HEGRA IACT system, was reported in 2002 [54, 55]. Subsequent observations by the IACT observatories MAGIC and VERITAS have confirmed the extension of the source and provided more precise measurements of its spectrum. All instruments find a power-law spectrum consistent with $\Gamma=2.1$, although there appears to be some disagreement between VERITAS and MAGIC as to the flux normalization $[56,57]$. No measurement thus far has found evidence for either an energy-dependent morphology of the source or a spectral cutoff up to 20 $\mathrm{TeV}$, although current measurements are not highly constraining in either case [56, 57].

Based on deep VERITAS observations of $\mathrm{TeV}$ J2032+4130, Aliu et al. [57] strongly suggest that TeV $\mathrm{J} 2032+4130$ is a relic PWN powered by the co-located gamma-ray pulsar PSR J2032+4127 [50]. Uncertainties in the estimate of the pulsar's distance raise questions 


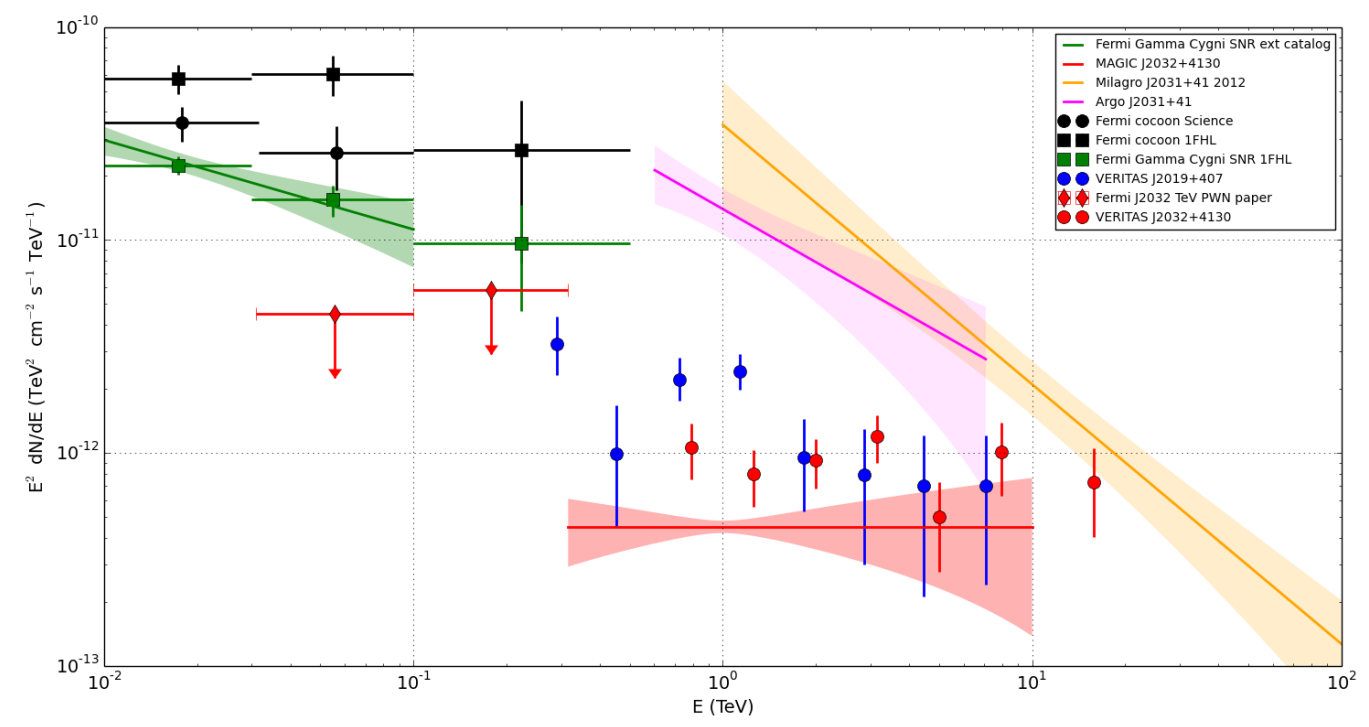

Figure 5: Potential contributions to the cocoon broadband spectrum: VER J2019+407 (red dots), TeV J2032+4130 (VERITAS, blue dots; MAGIC, red butterfly), the $\gamma$ Cygni SNR (1FHL [40] flux points, green squares; spectrum from [49], green butterfly) and MGRO J2032+4130 (yellow butterfly). Also shown: the ARGO J2032+41 spectrum (pink butterfly), the cocoon flux points ([37], black dots; [40], black squares), and Fermi LAT upper limits on the PWN of PSR J2032+4127 (red arrows). Figure courtesy Luigi Tibaldo. (A color version of this figure is available in the online journal).

about TeV J2032+4130's relationship to other structures in Cygnus-X. Application of standard Cordes and Lazio [58] models for dispersion in the Milky Way to recent radio observations [59] of the pulsar place PSR $\mathrm{J} 2032+4127$ at $3.6 \mathrm{kpc}$, beyond Cyg OB2. Alternative approaches still place it at $1.7 \mathrm{kpc}$, consistent with standard distance estimates for both Cyg OB2 and the $\gamma$ Cygni SNR [59]. On the grounds that few of the nearby massive OB stars actually overlap the observed VHE gamma-ray emission, Aliu et al. [57] disfavor a previously popular interpretation that $\mathrm{TeV} \mathrm{J} 2032+4130$ is powered by winds from OB stars in the Cyg OB2 association. However, the stellar wind hypothesis cannot be definitively ruled out.

\subsection{An Incomplete Picture}

Figures 3 and 5 neatly illustrate the challenge of pinning down a broadband spectrum for the Cygnus cocoon from the extant multiwavelength data. On the one hand, a simple power-law extrapolation of the Fermi $L A T$ spectrum to higher energies would agree with the MGRO J2031+41 spectrum. On the other, four sources seen below $10 \mathrm{TeV}$ potentially contribute to the emission from MGRO J2031+41: the cocoon, the $\gamma$ Cygni SNR, VER J2019+407, and TeV J2032+4130. There is no question that the cocoon and $\mathrm{TeV} \mathrm{J} 2032+4130$ contribute to the emission; the degree to which the $\gamma$ Cygni SNR and VER J2019+407 contribute is less clear. What is clear is that the spectrum of any source contributing to the emission from MGRO J2031+41 must cut off somewhere between $1 \mathrm{TeV}$ and $20 \mathrm{TeV}$. Power-law extrapolations of the VER J2019+407 and TeV J2032+4130 spectra would quickly overshoot the MGRO J2031+41 spectrum above $20 \mathrm{TeV}$; extrapolations of the cocoon and the $\gamma$ Cygni SNR spectra would likewise be impossible to accommodate.

Figure 5 includes the ARGO detection [13] for completeness. However, this detection is difficult to reconcile with the other observations as it has a flux level comparable to MGRO J2031+41 but an extent compatible with TeV J2032+4130.

\subsection{MGRO J2019+37 and Cyg OBI}

MGRO J2031+41 is not the only extended source seen by Milagro within Cygnus-X. MGRO J2019+37 is the brightest Milagro source in the Cygnus region, with a flux of about $80 \%$ of the Crab Nebula flux at $20 \mathrm{TeV}$ [44]. The bright core of the source has an extent of at least $1^{\circ}$. As with MGRO J2031+41, indications are that MGRO J2019+37 is a synthesis of 


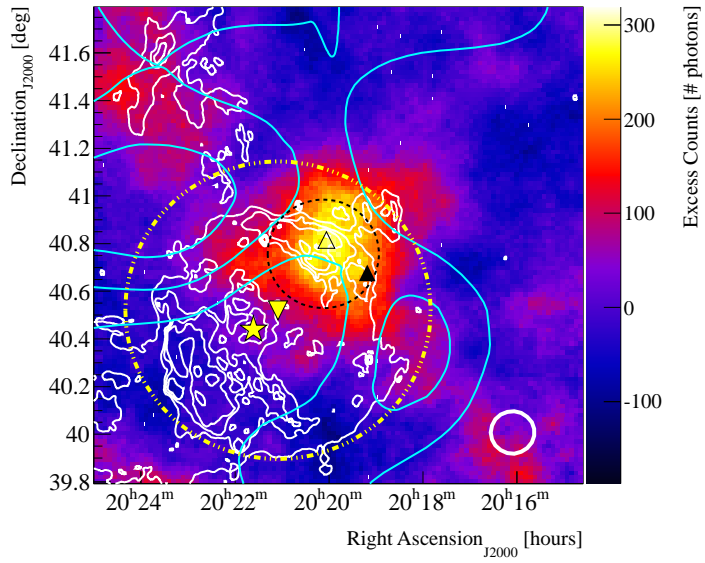

Figure 4: Background-subtracted gamma-ray counts map showing the VERITAS detection of VER J2019+407 and its fitted extent (black dashed circle). Canadian Galactic Plane Survey (CGPS) $1420 \mathrm{MHz}$ continuum radio contours at brightness temperatures of $23.6 \mathrm{~K}, 33.0 \mathrm{~K}, 39.6 \mathrm{~K}, 50 \mathrm{~K}$ and $100 \mathrm{~K}$ (white) [48] show the extent of the radio remnant. The star symbol shows the location of PSR J2021+4026. The fitted centroid and extent of the emission detected by Fermi LAT above $10 \mathrm{GeV}$ are indicated by the inverted triangle and dot-dashed circle (yellow). The open and filled triangles (black) show the positions of the Fermi LAT catalog sources 1FGL J2020.0+4049 and 2FGL J2019.1+4040, now subsumed into the extended $\mathrm{GeV}$ emission from the entire remnant [49]. The $0.16,0.24$, and 0.32 photons bin $^{-1}$ contours of the Fermi LAT detection of the Cygnus cocoon are shown in cyan [37]. The VERITAS gamma-ray PSF is shown for comparison (white circle, bottom right). Reproduced from [36]. (A color version of this figure is available in the online journal).

multiple gamma-ray sources, including multiple potential PWNe. Recent observations by VERITAS bear this out. Figure 6 shows VERITAS' current best picture of MGRO J2019+37 and its vicinity between 600 $\mathrm{GeV}$ and $10 \mathrm{TeV}$ [60]. VERITAS already distinguishes two sources in the region: the point-like source VER J2019+368 and VER J2019+368, an apparent ridge of diffuse emission $\sim 1^{\circ}$ in length, roughly bounded by the bright bubble HiI region Sh 2-104 to the west and the energetic gamma-ray and radio pulsar PSR J2021+3651 to the east. There appears to be a clear correspondence between VER J2016+371 and SNR CTB 87. CTB 87's radio morphology and the presence of pulsar candidate CXOU J201609.2+371110 within the radio contours support CTB 87's identification as a PWN [60]. Aliu et al. [60] also argue strongly for a relic PWN interpretation of VER J2016+371/CTB 87.

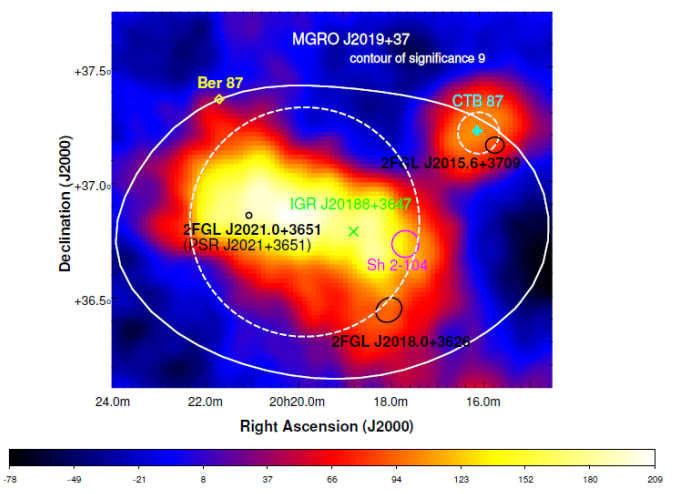

Figure 6: Map of the gamma-ray excess above $600 \mathrm{GeV}$, seen by VERITAS in the vicinity of MGRO J2019+37. The color bar indicates the number of excess events within a $0.23^{\circ}$ search radius. White dashed circles indicate the regions used to extract the spectra of VER J2016+371 and VER J2019+368. The $9 \sigma$ significance contour of MGRO J2019+37 is overlaid in solid white. The remaining solid ellipses, diamonds and crosses indicate the locations of potential counterparts. Reproduced from [60]. (A color version of this figure is available in the online journal).

A unique interpretation of VER J2019+368 and MGRO J2019+37 is more challenging. Figure 7 shows that the VER J2019+368 spectrum merges with that of MGRO J2019+37 at high energies. Taken together with the ARGO-YBJ upper limits, which agree well with the VERITAS spectrum, this argues for VER J2019+368 as the dominant contribution to MGRO J2019+37. However, VER J2019+368 itself likely incorporates emission from several unresolved sources. These may include the PWN of PSR J2021+3651 and the H II region Sh 2-104.

\section{The Next Chapter}

In the preceding sections, we considered a number of questions, including Geminga's potential contribution to the positron excess and the nature and origin of the accelerated particles in the Cygnus cocoon, that point towards a common wish list in terms of both new technology and new data analysis techniques. This wish list includes: improved angular resolution, particularly at multi-TeV energies, to reduce source confusion; improved sensitivity between $100 \mathrm{GeV}$ and 10 $\mathrm{TeV}$ to highly extended $\left(\sim 1^{\circ}-3^{\circ}\right)$ sources such as the 


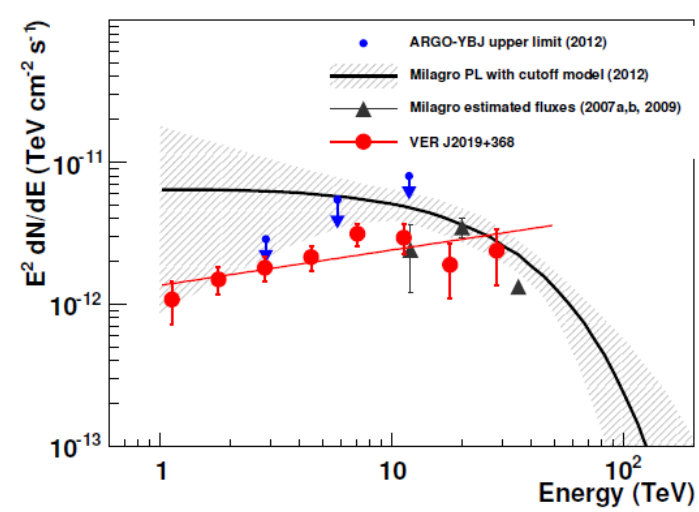

Figure 7: Spectral energy distribution of MGRO J2019+37 and VER J2019+368. The VER J2019+368 spectrum from $1 \mathrm{TeV}$ to almost $30 \mathrm{TeV}$ (red dots) is best fit with a power law with $\Gamma=1.75 \pm 0.3$. The original Milagro flux points are shown at 12,20 and $35 \mathrm{TeV}$ (black triangles) [61, 62, 43]. The black curve shows the 2012 best-fit spectrum for MGRO J2019+37: a power law with a cutoff [44]. The shadowed area corresponds to the $1 \sigma$ band. ARGO-YBJ $90 \%$ confidence-level upper limits for MGRO J2019+37 are shown with blue arrows [13]. Reproduced from [60]. (A color version of this figure is available in the online journal).

$\gamma$ Cygni SNR, the Geminga gamma-ray PWN and the Cygnus cocoon; more effective methods of combining spatial and spectral information from multiple instruments to constrain the accelerated populations responsible for gamma-ray emission; and order of magnitude improvements in detector effective area and sensitivity above $100 \mathrm{GeV}$.

In this section we primarily consider near-term advances in technology and data analysis methods with the potential to address the first three bullets of this wish list.

\section{1. $H A W C$}

The newly-commissioned High Altitude Water Cherenkov (HAWC) Observatory is a secondgeneration water Cherenkov instrument, sensitive to both gamma rays and cosmic rays between 100 $\mathrm{GeV}$ and $100 \mathrm{TeV}$ [16]. HAWC consists of 300 water Cherenkov detectors, deployed over an area of approximately 22,000 square meters. Each detector, a water tank with 4 large photomultiplier tubes, samples the energetic secondary particles that reach the ground from an atmospheric shower initiated by a gamma- or cosmic ray. The HAWC site is $4100 \mathrm{~m}$ above sea level in the mountains of central Mexico.

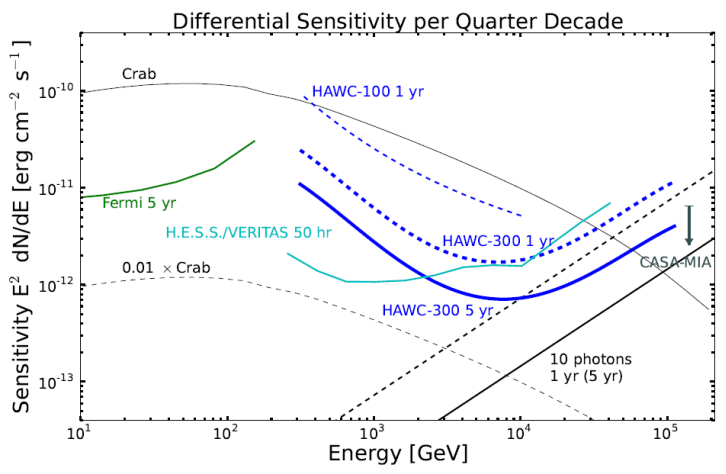

Figure 8: A comparison of the differential sensitivities of various construction stages of HAWC to those of Fermi LAT and typical IACT instruments. Reproduced from [16].

Figure 8 shows HAWC's differential sensitivity as a function of energy, compared to both Fermi LAT and current IACT instruments. Above $10 \mathrm{TeV}$, HAWC achieves angular resolution $\left(\sim 0.1^{\circ}\right)$ comparable to that of IACTs at $1 \mathrm{TeV}$ and that of Fermi LAT above $1 \mathrm{GeV}$. HAWC, which has angular resolution far superior to that of Milagro at all energies, will bring the sources seen by Milagro into sharper focus over a broader energy range. However, below $10 \mathrm{TeV}$ HAWC's angular resolution and sensitivity degrade sharply (HAWC's angular resolution is $\sim 0.4^{\circ}$ at $1 \mathrm{TeV}$ ). Since Fermi LAT's effective area begins to degrade past $10 \mathrm{GeV}$, this creates a "gap" in coverage between $100 \mathrm{GeV}$ and $10 \mathrm{TeV}$.

In the near term, this gap is best filled by IACTs such as VERITAS. Together, these three instruments can provide unbroken spectral coverage of regions such as Cygnus- $X$ and sources such as Geminga, with similar angular resolution, between $1 \mathrm{GeV}$ and $100 \mathrm{TeV}$. In order to take advantage of this treasure trove of data, however, we must develop data analysis methods that allow IACTs to grapple with sources comparable to or larger than the field of view. We consider such a method in the subsequent section.

\subsection{The (" $3 D$ ”) Maximum Likelihood Method}

As noted earlier, the most commonly used IACT data analysis methods perform poorly when presented with a source that fills a large fraction of the field of view. The classic technique of "on-off" observation compensates for this by pairing source observations with observations of a blank field, with the off-source field used to estimate the cosmic ray background level. This method is inefficient as the time spent in off-source observations 
reduces the available source exposure by at least a factor of two. Unless the ratio of off-source to on-source observations is kept much higher than two, this method also has diluted sensitivity compared to the reflectedregion and ring-background methods. By contrast, an innovative maximum likelihood method can achieve a point-source sensitivity comparable to that of the ringbackground model [63].

The unbinned maximum likelihood method discussed here is similar to that used by Fermi LAT. The "3D" unbinned maximum likelihood method (3D MLM) proposed for IACT instruments fits a multi-component model to the distribution of photons in both projected sky coordinates and a cosmic ray background rejection parameter. The inclusion of a background rejection parameter permits the fit to separate gamma-ray source and background components even when the spatial models of these components are indistinguishable [63]. The preliminary studies described in this paper use a well-understood and widely used background rejection parameter, mean scaled width (MSW) [64].

Probability density functions (PDFs) describe the distribution of the cosmic ray background and any potential gamma-ray sources in all three variables. The quantity minimized is $-2 \ln L$ where $L$ is the product of likelihoods (probabilities of obtaining the observed event data, given the adopted model) over sets of observations distinguished by key characteristics (e.g. the number of telescopes used in event reconstruction, the sky tracking position of the observation, the detector configuration, the zenith angle and the range of reconstructed photon energies). Each energy bin requires distinct source and background models derived from simulations and cosmic ray data. Finer energy binning improves sensitivity to spectral parameters but increases the volume of simulations and data needed to develop the models.

Models: The model for a given observation set $i$ (data category) takes the following form for the single-source case:

$$
\begin{array}{r}
\mathrm{F}_{i}(x, y, w)=N_{i}^{\gamma}\left(\tau, \alpha_{j}, A_{e f f}^{i}, S\right) S_{i}^{\gamma}(x, y) \\
\times M_{i}^{\gamma}(w)+N_{i}^{B}(\tau) S_{i}^{B}(x, y) \times M_{i}^{B}(w)
\end{array}
$$

with $S(x, y)$ and $M(w)$ describing the signal and background spatial and MSW distributions, respectively. The approximation of the three-dimensional PDF by a product of PDFs is valid in the limit where the spatial and MSW distributions are uncorrelated. The predicted number of events $N_{i}^{\gamma}$ (the number of source photons in a given data category) is a function of the following quantities: the livetime of the observation, $\tau$, the source spectral parameters $\alpha_{j}$, and the effective area of the instru- ment, $A_{e f f}$. $A_{e f f}$ describes the probability of detecting a given photon as a function of photon energy and radial offset within the camera, the variations in flux intensity across the surface of the source, the position of the source within the camera, and the fraction of the source contained within the field of view. It also has a conditional dependence on observation characteristics such as sky brightness, zenith angle, and azimuth angle.

The number and nature of the parameters $\alpha_{j}$ depend on the source spectral model: e.g. for a power law, $d N / d E=f_{0}\left(E / E_{0}\right)^{-\Gamma}$, the $\alpha_{j}$ would be $f_{0}$ (differential source flux at a reference energy) and $\Gamma$ (spectral index), while a power-law model with an exponential cutoff would add a third parameter for the cutoff energy. As the background models are ad hoc, derived from cosmic ray data and simulations, the background parameter $N_{i}^{B}$ has a relatively straightforward dependence on the livetime, with a conditional dependence on the observation characteristics cited above. An extended maximum likelihood is used, which accounts for Poisson fluctuations in the total number of events for each category.

Simultaneous fitting: Since the full log-likelihood is merely the sum of the individual log-likelihoods, the data categories may be fit simultaneously. In a simultaneous fit a separate model, carefully matched in terms of response functions, etc., is used for each data category. In principle, if each category in the fit had a completely separate set of free parameters, the subdivision of the data into categories would lead to a noticeable loss of statistical power. However, the source models ultimately depend on the same physical parameters that are used to describe the source morphology and spectrum and these parameters are constrained to be the same across all categories. For instance, a source modeled as a simple Gaussian in photon arrival direction with a power-law spectrum would have three key parameters: the width of the Gaussian, $f_{0}$ and $\Gamma$. This preserves the statistical power of the fit. The individual background normalization parameters $N_{i}^{B}$ may be left as individual free parameters in the fit or constrained to a smaller number of free parameters as appropriate.

\subsection{An Illustrative Test Case}

We illustrate the capabilities of the 3D MLM using simulated observations of the Cygnus cocoon region. This region contains multiple overlapping sources with radial extensions ranging from a few tenths of a degree to two degrees, all of which are either detected or have the potential to be detected at energies greater than 300 $\mathrm{GeV}$. Out of all the scientifically interesting regions considered in this paper, the cocoon and environs therefore 
present the single most complete test of the 3D MLM's capabilities.

The simulations used here are simple toy model simulations based on the expected distribution of both signal and background in the three dimensions (spatial and MSW) of the fit. They assume a VERITAS-like instrument with comparable sensitivity and identical field of view. The study uses approximately 200 hours of simulated observations tiled in a non-ideal, irregular exposure pattern. The version of the 3D MLM used in these studies is a less sophisticated version of the fit described in the preceding section. In this case the spectral behavior of the sources is not left as a free parameter and less sophisticated models are used for the instrument response functions. Due to a number of factors, including the time required to develop reasonable background models in each bin of energy, this fit uses a single pair of source and background models, applicable between $500 \mathrm{GeV}$ and $1 \mathrm{TeV}$. This focuses the study on an energy range where Fermi LAT provides no data, excludes energies for which the spectral behavior of $\mathrm{TeV} \mathrm{J} 2032+4130$ is poorly known and excludes energies above $1 \mathrm{TeV}$ where extrapolations of the source spectra grow most uncertain.

The simulation incorporates both of the known gamma-ray sources detected by VERITAS (TeV J2032+4130 and VER J2019+407) and assumes the spectral parameters and spatial extensions reported by VERITAS for these sources. Simple power-law extrapolations are used for the cocoon and $\gamma$ Cygni, based on the spectral parameters and fluxes reported in [37], [49], and the 1FHL catalog [40]. The spatial model used in all cases is a two-dimensional Gaussian of the appropriate extent. The gamma-ray pulsars are not expected to contribute significantly above $500 \mathrm{GeV}$ and are therefore omitted from the simulations. In these preliminary simulations we have also neglected the contribution from the Galactic diffuse emission. The Galactic diffuse spectrum falls off rapidly relative to that of any of the sources of interest and the contribution at any given point in the observed region is likely to be below the sensitivity threshold of VERITAS.

The square root of the test-statistic, $T S=$ $-2 \ln \left(L_{0} / L_{1}\right)$ where $L_{1}$ is the maximum likelihood of the test hypothesis and $L_{0}$ that of the null hypothesis, is used as a proxy for detection significance. The 3D MLM fit detects emission from the simulated cocoon and simulated $\gamma$ Cygni with $\sqrt{T S}$ values of greater than 45 and 8, respectively. Figures 9 and 10 also show that the $\gamma$ Cygni and cocoon morphologies emerge clearly. The fit does a reasonable job of disentangling the overlapping sources of gamma-ray emission, with one excep-

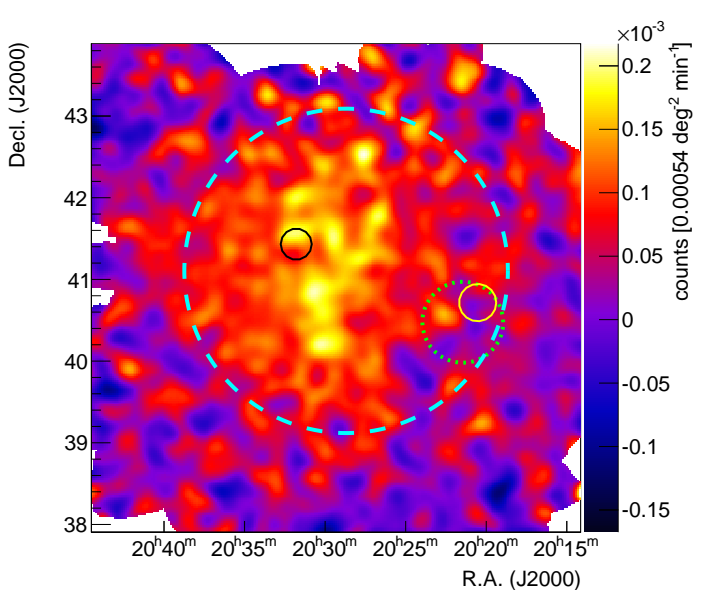

Figure 9: Smoothed residual map (data minus background model), derived from the 3D MLM applied to a toy model simulation of $\sim 200$ hours of irregularly tiled observations by a VERITAS-like observatory. 1- $\sigma$ contours are shown for the Gaussian source models: the cocoon (dashed blue), $\gamma$ Cygni SNR (short-dashed green), VER J2019+407 (yellow), and TeV J2032+4130 (black). The latter three sources are included in the background model in this case. (A color version of this figure is available in the online journal).

tion. VER J2019+407 can only be distinguished from the $\gamma$ Cygni SNR emission at the $3 \sigma$ level. It is interesting to note that this particularly challenging case of source confusion parallels the evolution of Fermi LAT's perspective on the SNR. A source co-located with VER J2019+407, reported in the 1FGL and 2FGL catalogs, was subsequently subsumed into the disk-like emission from the entire remnant [49].

It should also be noted that this study makes somewhat pessimistic predictions. While a simple twodimensional Gaussian was used to describe the cocoon in [37], this assumed morphology is almost certainly more featureless and diffuse than the actual cocoon morphology, which should therefore be easier to resolve. Furthermore, these sources are likely to develop distinct cutoffs at higher energies. A more complete fit that leaves spectral parameters free and covers the energy range from a few hundred $\mathrm{GeV}$ to $10 \mathrm{TeV}$ should provide additional sensitivity and improve the fit's ability to distinguish overlapping sources in this region.

\subsection{Future Instruments}

Future studies of the type discussed here will not be limited to the combined capabilities of Fermi LAT IACTs, and HAWC. The Cherenkov Telescope Array 


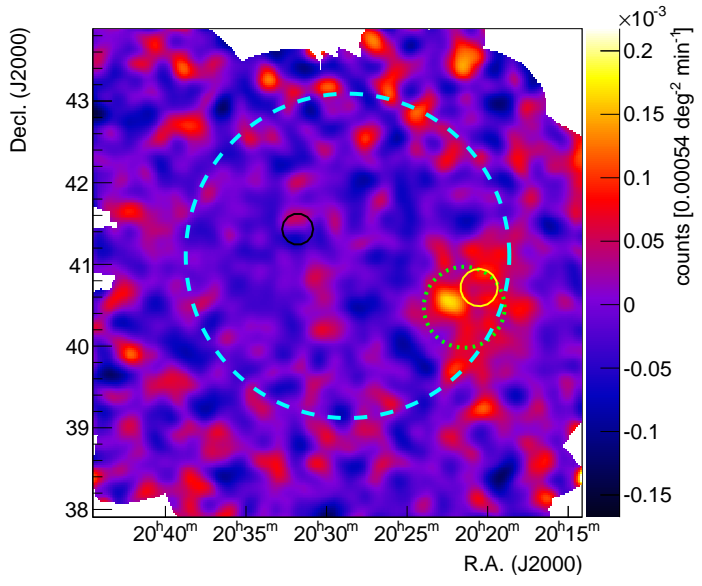

Figure 10: Smoothed residual map from the same simulated scenario as in Figure 9, but with all sources but $\gamma$ Cygni included in the background model. (A color version of this figure is available in the online journal).

(CTA), a pair of planned next-generation IACT arrays in the northern and southern hemispheres, promises broader energy coverage, dramatically improved $(<$ $\left.0.04^{\circ}\right)$ angular resolution, a comparatively large (7 $8^{\circ}$ ) field of view and order-of-magnitude increase in sensitivity between $100 \mathrm{GeV}$ and $10 \mathrm{TeV}$ [65, 66]. LHAASO, a hybrid instrument combining water Cherenkov and particle detectors [67], also promises unprecedented sensitivity between $20 \mathrm{TeV}$ and a PeV.

\section{Conclusions}

Pulsars and their associated PWNe may play a role in explaining the unexpected increase in cosmic ray positrons at high energies. In particular, studies of the Geminga PWN at very high energies may constrain the scenario in which a single local source accounts for the entire positron excess. PWNe also complicate the study of gamma-ray emission from potential cosmic ray nurseries. Harnessing the combined power of data from Fermi LAT, IACTs such as VERITAS, and HAWC to address these questions presents unique but not insurmountable technical challenges. In the immediate future, combined studies using current-generation instruments and new data analysis techniques will shed new light on the relationship of PWNe to the larger cosmic ray mystery story. What we learn now will guide even more sensitive studies with powerful next-generation instruments over the coming decades.

\section{Acknowledgments}

A. Weinstein thanks L. Tibaldo, S. Casanova, L. Grenier, and P. Majumdar for helpful discussions of of the Cygnus cocoon scenario. The VERITAS 3D MLM development has been supported in part by NASA grant NNX11A086G. VERITAS is supported by grants from the U.S. Department of Energy Office of Science, the U.S. National Science Foundation and the Smithsonian Institution, by NSERC in Canada, and by Science Foundation Ireland (SFI 10/RFP/AST2748). We acknowledge the excellent work of the technical support staff at the Fred Lawrence Whipple Observatory and at the collaborating institutions in the construction and operation of the instrument.

\section{References}

[1] V. Hess, Über Beobachtungen der durchdringenden Strahlung bei sieben Freiballonfahrten, Physikalische Zeitschrift 13 (1912) 1084-1091.

[2] D. Pacini, Penetrating radiation at the surface of and in water, Nuovo Cimento 3 (1912) 93

[3] W. R. Binns, M. E. Wiedenbeck, M. Arnould, et al., OB Associations, Wolf Rayet Stars, and the Origin of Galactic Cosmic Rays, Space Sci. Rev. 130 (2007) 439-449. arXiv : 0707 .4645, doi : 10.1007/s11214-007-9195-1.

[4] M. Aguilar, G. Alberti, B. Alpat, et al., First result from the alpha magnetic spectrometer on the international space station: Precision measurement of the positron fraction in primary cosmic rays of 0.5-350 gev, Phys. Rev. Lett. 110 (2013) 141102. doi:10.1103/PhysRevLett.110.141102.

[5] M. Ackermann, M. Ajello, A. Allafort, et al., Measurement of Separate Cosmic-Ray Electron and Positron Spectra with the Fermi Large Area Telescope, Phys. Rev. Lett. 108 (1) (2012) 011103. arXiv:1109.0521, doi:10.1103/PhysRevLett. 108.011103.

[6] J. Chang, J. H. Adams, H. S. Ahn, et al., An excess of cosmic ray electrons at energies of 300-800GeV, Nature 456 (2008) $362-$ 365. doi:10.1038/nature 07477 .

[7] S. W. Barwick, J. J. Beatty, C. R. Bower, et al., The Energy Spectra and Relative Abundances of Electrons and Positrons in the Galactic Cosmic Radiation, ApJ 498 (1998) 779-789. arXiv:astro-ph/9712324, doi:10.1086/305573.

[8] M. Boezio, G. Barbiellini, V. Bonvicini, et al., Measurements of cosmic-ray electrons and positrons by the Wizard/CAPRICE collaboration, Advances in Space Research 27 (2001) 669-674. doi : 10.1016/S0273-1177(01)00108-9.

[9] K. Yoshida, S. Torii, T. Yamagami, et al., Cosmic-ray electron spectrum above $100 \mathrm{GeV}$ from PPB-BETS experiment in Antarctica, Advances in Space Research 42 (2008) 1670-1675. doi:10.1016/j.asr.2007.04.043

[10] F. Aharonian, A. G. Akhperjanian, G. Anton, et al., Probing the ATIC peak in the cosmic-ray electron spectrum with H.E.S.S., A\&A 508 (2009) 561-564. arXiv : 0905.0105, doi : 10.1051/0004-6361/200913323.

[11] M. Di Mauro, F. Donato, N. Fornengo, et al., Interpretation of AMS-02 electrons and positrons data, JCAP 4 (2014) 6. arXiv : 1402.0321, doi:10.1088/1475-7516/2014/04/006. 
[12] J. A. Hinton, W. Hofmann, Teraelectronvolt Astronomy, Annual Review of A\&A 47 (2009) 523-565. arXiv: 1006.5210, doi : 10.1146/annurev-astro-082708-101816.

[13] B. Bartoli, P. Bernardini, X. J. Bi, et al., Observation of TeV Gamma Rays from the Cygnus Region with the ARGO-YBJ Experiment, ApJ 745 (2012) L22. arXiv: 1201.1973, doi: $10.1088 / 2041-8205 / 745 / 2 / L 22$

[14] A. A. Abdo, B. Allen, T. Aune, et al., A Measurement of the Spatial Distribution of Diffuse TeV Gamma-Ray Emission from the Galactic Plane with Milagro, ApJ 688 (2) (2008) 1078-1083.

[15] A. A. Abdo, B. Allen, T. Aune, et al., Discovery of Localized Regions of Excess 10-TeV Cosmic Rays, Phys. Rev. Lett. 101 (22) (2008) 221101. arXiv:0801.3827, doi:10.1103/PhysRevLett.101.221101.

[16] A. U. Abeysekara, R. Alfaro, C. Alvarez, et al., Sensitivity of the high altitude water Cherenkov detector to sources of multi-TeV gamma rays, Astroparticle Physics 50 (2013) 2632. arXiv: 1306.5800 , doi:10.1016/j.astropartphys. 2013.08 .002$.

[17] J. Holder, VERITAS: Status and Highlights, International Cosmic Ray Conference 12 (2011) 137. arXiv:1111.1225, doi: 10.7529/ICRC2011/V12/H11.

[18] O. Adriani, G. C. Barbarino, G. A. Bazilevskaya, et al., An anomalous positron abundance in cosmic rays with energies 1.5-100GeV, Nature 458 (2009) 607-609. arXiv : 0810.4995, doi:10.1038/nature07942.

[19] A. A. Abdo, M. Ackermann, M. Ajello, et al., Measurement of the Cosmic Ray $\mathrm{e}^{+}+\mathrm{e}^{-}$Spectrum from $20 \mathrm{GeV}$ to $1 \mathrm{TeV}$ with the Fermi Large Area Telescope, Phys. Rev. Lett. 102 (18) (2009) 181101. arXiv:0905.0025, doi:10.1103/PhysRevLett. 102.181101.

[20] D. Hooper, P. Blasi, P. Dario Serpico, Pulsars as the sources of high energy cosmic ray positrons, Journal of Cosmology and Astroparticle Physics 1 (2009) 25. arXiv:0810.1527, doi: 10.1088/1475-7516/2009/01/025.

[21] V. Barger, Y. Gao, W.-Y. Keung, et al., Dark matter and pulsar signals for Fermi LAT, PAMELA, ATIC, HESS and WMAP data, Phys. Rev. B 678 (2009) 283-292. arXiv:0904.2001, doi:10.1016/j.physletb.2009.06.030.

[22] G. G. Pavlov, D. Sanwal, V. E. Zavlin, The Pulsar Wind Nebula of the Geminga Pulsar, ApJ 643 (2006) 1146-1150. arXiv: astro-ph/0511364, doi:10.1086/503250.

[23] H. Yüksel, M. D. Kistler, T. Stanev, TeV Gamma Rays from Geminga and the Origin of the GeV Positron Excess, Phys. Rev. Lett. 103 (5) (2009) 051101. arXiv:0810.2784, doi:10.1103/PhysRevLett.103.051101.

[24] F. Acero, M. Ackermann, M. Ajello, et al., Constraints on the Galactic Population of TeV Pulsar Wind Nebulae Using Fermi Large Area Telescope Observations, ApJ 773 (2013) 77. arXiv: 1306.5735, doi:10.1088/0004-637X/773/1/77.

[25] D. Kieda, Search for TeV Emission from Geminga by the VERITAS Observatory, in: F. A. Aharonian, W. Hofmann, F. Rieger (Eds.), American Institute of Physics Conference Series, Vol. 1085 of American Institute of Physics Conference Series, 2008, pp. 269-272. doi:10.1063/1.3076657.

[26] G. M. Finnegan, High energy gamma-ray astronomy observations of Geminga with the VERITAS array, Ph.D. thesis, The University of Utah (2012).

[27] D. Berge, S. Funk, J. Hinton, Background modelling in very-high-energy $\gamma$-ray astronomy, A\&A 466 (2007) 12191229. arXiv:arXiv:astro-ph/0610959, doi:10.1051/ 0004-6361:20066674.

[28] F. Aharonian, A. G. Akhperjanian, A. R. Bazer-Bachi, et al., First detection of a VHE gamma-ray spectral maximum from a cosmic source: HESS discovery of the Vela $\mathrm{X}$ nebula,
A\&A 448 (2006) L43-L47. arXiv:astro-ph/0601575, doi : 10.1051/0004-6361:200600014.

[29] A. Abramowski, F. Acero, F. Aharonian, et al., Probing the extent of the non-thermal emission from the Vela $X$ region at TeV energies with H.E.S.S., A\&A 548 (2012) A38. arXiv: 1210.1359, doi:10.1051/0004-6361/201219919.

[30] W. Bednarek, M. Bartosik, Cosmic rays from Galactic pulsars, A\&A 423 (2004) 405-413. arXiv : astro-ph/0405310, doi : 10.1051/0004-6361:20047005.

[31] D. Horns, F. Aharonian, A. I. D. Hoffmann, A. Santangelo, Nucleonic gamma-ray production in pulsar wind nebulae, Ap\&SS 309 (2007) 189-195. arXiv : astro-ph/0609386, doi: 10.1007/s10509-007-9444-5.

[32] W. Bednarek, High energy processes in pulsar wind nebulae, Ap\&SS 309 (2007) 179-187. arXiv:astro-ph/0610307, doi:10.1007/s10509-007-9396-9.

[33] O. Tibolla, New developments in the ancient Pulsar Wind Nebulae scenario., International Cosmic Ray Conference 6 (2011) 202. arXiv:1109.3144, doi:10.7529/ICRC2011/ V06/1233.

[34] M. J. Vorster, O. Tibolla, S. E. S. Ferreira, et al., Timedependent Modeling of Pulsar Wind Nebulae, ApJ 773 (2013) 139. arXiv: 1309.7137 , doi : $10.1088 / 0004-637 \mathrm{X} / 773 / 2 /$ 139.

[35] A. Haungs, H. Rebel, M. Roth, Energy spectrum and mass composition of high-energy cosmic rays, Reports on Progress in Physics 66 (7) (2003) 1145.

URL http://stacks.iop.org/0034-4885/66/i=7/a= 202

[36] E. Aliu, S. Archambault, T. Arlen, et al., Discovery of TeV Gamma-Ray Emission toward Supernova Remnant SNR G78.2+2.1, ApJ 770 (2013) 93. arXiv:1305.6508, doi: 10.1088/0004-637X/770/2/93.

[37] M. Ackermann, M. Ajello, A. Allafort, et al., A Cocoon of Freshly Accelerated Cosmic Rays Detected by Fermi in the Cygnus Superbubble, Science 334 (6059) (2011) 1103-1107.

[38] J.-M. Le Duigou, J. Knödlseder, Characteristics of new star cluster candidates in the Cygnus area, A\&A 392 (2002) 869-884. doi: 10.1051/0004-6361:20020984.

[39] A. Konopelko, R. W. Atkins, G. Blaylock, et al., Observations of the Unidentified TeV $\gamma$-Ray Source TeV J2032+4130 with the Whipple Observatory 10 m Telescope, ApJ 658 (2007) 10621068. arXiv: astro-ph/0611730, doi:10.1086/511262.

[40] The Fermi-LAT Collaboration, The First Fermi-LAT Catalog of Sources Above $10 \mathrm{GeV}$, ArXiv e-printsarXiv: 1306.6772

[41] L. A. Higgs, T. L. Landecker, R. S. Roger, The true extent of the Gamma Cygni supernova remnant, AJ 82 (1977) 718-724. doi: $10.1086 / 112114$.

[42] T. A. Lozinskaya, V. V. Pravdikova, A. V. Finoguenov, The Supernova Remnant G78.2+2.1: New Optical and X-ray Observations, Astronomy Letters 26 (2000) 77-87. doi:10.1134/1. 20371.

[43] A. A. Abdo, B. T. Allen, T. Aune, et al., Milagro Observations of Multi-TeV Emission from Galactic Sources in the Fermi Bright Source List, ApJ 700 (2009) L127-L131. arXiv : 0904.1018, doi: 10.1088/0004-637X/700/2/L127.

[44] A. A. Abdo, U. Abeysekara, B. T. Allen, et al., Spectrum and Morphology of the Two Brightest Milagro Sources in the Cygnus Region: MGRO J2019+37 and MGRO J2031+41, ApJ 753 (2012) 159. arXiv:1202.0846, doi:10.1088/ 0004-637X/753/2/159.

[45] X. Zhang, Y. Zheng, T. L. Landecker, L. A. Higgs, Multifrequency radio spectral studies of the supernova remnant $G$ 78.2+2.1., A\&A 324 (1997) 641-650.

[46] Y. Uchiyama, T. Takahashi, F. A. Aharonian, J. R. Mattox, 
ASCA View of the Supernova Remnant $\gamma$ Cygni (G78.2+2.1): Bremsstrahlung X-Ray Spectrum from Loss-flattened Electron Distribution, ApJ 571 (2002) 866-875. arXiv:astro-ph/ 0202414, doi: $10.1086 / 340121$

[47] T. L. Landecker, R. S. Roger, L. A. Higgs, Atomic hydrogen in a field in Cygnus $\mathrm{X}$ containing the supernova remnant G78.2+2.1, A\&AS 39 (1980) 133-151.

[48] A. R. Taylor, S. J. Gibson, M. Peracaula, et al., The Canadian Galactic Plane Survey, AJ 125 (2003) 3145-3164. doi:10. 1086/375301.

[49] J. Lande, M. Ackermann, A. Allafort, et al., Search for Spatially Extended Fermi Large Area Telescope Sources Using Two Years of Data, ApJ 756 (2012) 5. arXiv:1207.0027, doi : 10.1088/0004-637X/756/1/5.

[50] A. A. Abdo, M. Ackermann, M. Ajello, et al., The First Fermi Large Area Telescope Catalog of Gamma-ray Pulsars, ApJS 187 (2010) 460-494. arXiv:0910.1608, doi:10. 1088/0067-0049/187/2/460

[51] P. L. Nolan, A. A. Abdo, M. Ackermann, et al., Fermi Large Area Telescope Second Source Catalog, ApJS 199 (2012) 31. arXiv:1108.1435, doi:10.1088/0067-0049/199/2/31.

[52] L. Trepl, C. Y. Hui, K. S. Cheng, et al., Multiwavelength properties of a new Geminga-like pulsar: PSRJ2021+4026, MN RAS 405 (2010) 1339-1348. arXiv:1008.4313, doi:10. $1111 / j .1365-2966.2010 .16555 . x$.

[53] D. A. Leahy, K. Green, S. Ranasinghe, X-ray and radio observations of the $\gamma$ Cygni supernova remnant G78.2+2.1, MNRAS 436 (2013) 968-977. arXiv: 1307.3151, doi : 10.1093/ mnras/stt 1596 .

[54] F. Aharonian, A. Akhperjanian, M. Beilicke, et al., An unidentified $\mathrm{TeV}$ source in the vicinity of Cygnus OB2, A\&A 393 (2002) L37-L40. arXiv:astro-ph/0207528, doi:10. 1051/0004-6361:20021171.

[55] F. Aharonian, A. Akhperjanian, M. Beilicke, et al., The unidentified TeV source (TeV J2032+4130) and surrounding field: Final HEGRA IACT-System results, A\&A 431 (2005) 197-202. doi : 10.1051/0004-6361:20041552.

[56] J. Albert, E. Aliu, H. Anderhub, et al., MAGIC Observations of the Unidentified $\gamma$-Ray Source TeV J2032+4130, ApJ 675 (2008) L25-L28. arXiv:0801 . 2391, doi : 10 .1086/529520.

[57] E. Aliu, T. Aune, B. Behera, et al., Observations of the unidentified gamma-ray source TeV J2032+4130 by VERITAS, ArXiv e-printsarXiv: 1401.2828.

[58] J. M. Cordes, T. J. W. Lazio, NE2001.I. A New Model for the Galactic Distribution of Free Electrons and its Fluctuations, ArXiv Astrophysics e-printsarXiv : astro-ph/0207156.

[59] F. Camilo, P. S. Ray, S. M. Ransom, et al., Radio Detection of LAT PSRs J1741-2054 and J2032+4127: No Longer Just Gamma-ray Pulsars, ApJ 705 (2009) 1-13. arXiv : 0908. 2626. doi:10.1088/0004-637X/705/1/1.

[60] E. Aliu, T. Aune, B. Behera, et al., Spatially Resolving the Very High Energy Emission from MGRO J2019+37 with VERITAS, ApJ 788 (2014) 78. doi : 10.1088/0004-637X/788/1/78.

[61] A. A. Abdo, B. Allen, D. Berley, et al., Discovery of TeV Gamma-Ray Emission from the Cygnus Region of the Galaxy, ApJ 658 (2007) L33-L36. arXiv: astro-ph/0611691, doi : $10.1086 / 513696$.

[62] A. A. Abdo, B. Allen, D. Berley, et al., TeV Gamma-Ray Sources from a Survey of the Galactic Plane with Milagro, ApJ 664 (2007) L91-L94. arXiv : 0705.0707, doi : 10.1086/ 520717.

[63] J. V. Cardenzana, for the VERITAS Collaboration, VER J2019+407 and the Cygnus Cocoon, ArXiv e-printsarXiv: 1308.0055.

[64] A. Daum, G. Hermann, M. Heß, et al., First results on the per- formance of the HEGRA IACT array, Astroparticle Physics 8 (1997) 1-11. doi:10.1016/S0927-6505(97) 00031-5.

[65] V. A. Acciari, E. Aliu, T. Arlen, et al., Observation of Extended Very High Energy Emission from the Supernova Remnant IC 443 with VERITAS, ApJ 698 (2009) L133-L137. arXiv: 0905.3291, doi:10.1088/0004-637X/698/2/L133.

[66] M. Actis, G. Agnetta, F. Aharonian, et al., Design concepts for the Cherenkov Telescope Array CTA: an advanced facility for ground-based high-energy gamma-ray astronomy, Experimental Astronomy 32 (2011) 193-316. arXiv: 1008.3703, doi: 10 . 1007/s10686-011-9247-0.

[67] Y. Chen, B. Jiang, Multi-wavelength studies on interaction of supernova remnants with molecular clouds, Scientia Sinica Physica, Mechanica \& Astronomica 43 (2013) 1. arXiv: 1305. 3991, doi : 10.1360/132012-812. 
Available online at www.sciencedirect.com $\because \because$ ScienceDirect

Nuclear Physics B Proceedings Supplement 00 (2019) 1-1
Nuclear Physics B

Proceedings

Supplement

\section{Abstract \\ Keywords:}

1. 